\title{
Time-resolved RHEED Studies of the Growth of Epitaxial ZnSe Films on GaAs By Pulsed Laser Deposition
}

\section{Citation}

McCamy, James W. and Michael J. Aziz. 1997. Time-resolved RHEED studies of the growth of epitaxial ZnSe films on GaAs by pulsed laser deposition. Materials Research Society Symposium Proceedings 441: 621-626.

\section{Published Version}

http://www.mrs.org/s_mrs/sec.asp?CID=1646\&DID=8855

\section{Permanent link}

http://nrs.harvard.edu/urn-3:HUL.InstRepos:2798832

\section{Terms of Use}

This article was downloaded from Harvard University's DASH repository, and is made available under the terms and conditions applicable to Other Posted Material, as set forth at http:// nrs.harvard.edu/urn-3:HUL.InstRepos:dash.current.terms-of-use\#LAA

\section{Share Your Story}

The Harvard community has made this article openly available.

Please share how this access benefits you. Submit a story.

\section{Accessibility}




\title{
Time-resolved RHEED Studies of the Growth of Epitaxial ZnSe Films on GaAs By Pulsed Laser Deposition
}

James W. McCamy and Michael J. Aziz

Division of Engineering and Applied Sciences

Harvard University, Cambridge, MA 02138

\begin{abstract}
Film growth consists of two basic processes, deposition and surface relaxation, with opposing effects on the evolution of surface roughness. The pulsed-laser deposition (PLD) growth process has the unique feature of having periods of very high deposition rates on $\mu$ s time scales followed by periods, on the order of seconds, with only surface relaxation. In this paper we report the first efforts towards exploiting this unique feature to study these two basic processes independently. Thin epitaxial films of ZnSe were grown using PLD on (001) GaAs and $2^{\circ}$ miscut (001) GaAs substrates. For growth on both the singular and vicinal surfaces, RHEED patterns taken following growth showed clear, streaky first zone and sharp second zone spots, and well-defined Kikuchi lines; these features are indicative of a smooth growth surface and high quality film. No reconstruction of the growth surface was observed, in contrast to behavior observed in molecular beam epitaxy. Time-resolved RHEED measurements show that a single morphology developed during growth on singular (001) GaAs. However, during growth on miscut (001) GaAs, two morphologies developed, one transitory and one appearing to evolve towards steady state. When growth on the miscut substrate was stopped, recovery of the RHEED signal was observed. The rate of recovery could be attributed to two relaxation processes, as differentiated by their time constants. Potential origins of these observations are discussed.
\end{abstract}

\section{INTRODUCTION}

Epitaxial growth from the vapor phase requires the ordering of randomly deposited species onto crystalline lattice sites at the surface. This rearrangement of atoms occurs primarily by surface diffusion and proceeds by one of several growth modes, e.g., island growth (3-D), layerby-layer (2-D), or (on miscut surfaces) step-flow growth. These have been studied extensively under near-equilibrium conditions during molecular beam epitaxy (MBE) of semiconductor thin films [1]. PLD also can be used to grow epitaxial semiconductor films [2], and it is has been shown that, in some cases, these films are structurally comparable or superior to those grown by MBE [3]. This technique has also been used to grow p-type doped ZnTe films with hole concentrations equaling the maximum obtained by any technique [4]. Further, Chergui et al. [5] showed that the surface morphology of ZnSe films grown on (001) GaAs by PLD is smoother than that grown by metal-organic vapor phase epitaxy. These results are perhaps surprising because PLD is an energetic, far-from-equilibrium process, in which the kinetic energies of the depositing species are in the range $\mathrm{E}_{\mathrm{k}}=1-100 \mathrm{eV}$ [6]. The potential effects of deposition at these energies would include the breaking of surface reconstructions, an increase in surface diffusion, embedding of the depositing atoms into the surface layer, and the creation of bulk point defects.

Another primary difference between crystal growth in MBE and crystal growth in PLD is the instantaneous deposition rate. In MBE, the growth is continuous, such that the deposition flux is random in time and space, resulting in surface roughening as this "white noise" accumulates; kinetic competition from a variety of smoothening mechanisms determines the observed growth mode and the resulting surface structure. In contrast, PLD deposition involves periods of very high deposition on $\mu$ s timescales, separated by long time intervals with no deposition flux and during which only surface relaxation occurs. Thus, the deposition flux in PLD is random in space but periodic in time. This feature of PLD allows the deposition processes and surface relaxation processes to operate virtually independently.

One aspect of film growth in which one would expect these features to manifest themselves is in the mode of growth. It has been reported [7-10] that the PLD growth of oxides at high temperatures $\left(\sim 700^{\circ} \mathrm{C}\right)$ occurs primarily by layer-by-layer growth. Our work is the first reported investigation of the growth mode for the PLD growth of semiconductor films. 


\section{EXPERIMENTAL}

Substrates of (001) GaAs and (001) GaAs miscut $2^{\circ}$ towards the (110) were degreased and then etched (15s) in concentrated $\mathrm{H}_{2} \mathrm{SO}_{4}$. They were then mounted on the substrate heater with indium, and introduced into the growth chamber $\left(5 \times 10^{-9}\right.$ Torr base pressure $)$. Immediately before film growth, the substrates were heated to $570^{\circ} \mathrm{C}$ until the surface oxide desorbed, as determined by the appearance of well-defined reflection high energy electron diffraction (RHEED) spots, and then cooled to the growth temperature. In previous work it was determined that the optimum temperature for PLD growth of $\mathrm{ZnSe}$ on $\mathrm{GaAs}$ is $300^{\circ} \mathrm{C}$ [11]; thus, in this work we maintained the substrate growth temperature of $\mathrm{Tg}=300^{\circ} \mathrm{C}$.

A pulsed $\mathrm{KrF}(248 \mathrm{~nm})$ excimer laser beam ( $35 \mathrm{~ns}$ FWHM pulse duration) was passed through an aperture and brought to a focus on a $2.54 \mathrm{~cm}$-diameter polycrystalline ZnSe target (Plasmaterials, 99.999+\% purity) using a single $+200 \mathrm{~cm}$ spherical lens. The data reported here were obtained using an estimated energy density at the target of $\mathrm{E}_{\mid}=1.67 \mathrm{~J} / \mathrm{cm}^{2}$ and a targetsubstrate separation of $\sim 15 \mathrm{~cm}$. The film growth rate was monitored in situ from interference oscillations in the intensity of a HeNe $(633 \mathrm{~nm})$ laser beam reflected from the upper and lower surfaces of the growing film. For the growth conditions given here, typical growth rates were in the range of $0.04-0.06 \mathrm{~nm}$ per laser pulse.

The structure of the surface was monitored during and following growth using RHEED. The e-beam source for the RHEED was a $20 \mathrm{keV}$ unit (Staib Instruments). The data was acquired by monitoring the spot intensity on a phosphorescent screen using a Peltier cooled CCD camera and related acquisition equipment (K-Space Associates). For time-resolved acquisition, the acquisition time for each data point was $33 \mathrm{~ms}$. At times, the laser firing and camera timing overlapped, so that the UV light from the laser interacted with the RHEED screen. These points were discarded with no loss of information regarding the growth process. To remove additional systemic noise, the time-resolved data were normalized with respect to the background signal close to the diffraction signal of interest.

\section{RESULTS - GROWTH ON SINGULAR SURFACES}

Qualitative information about the quality of the growth surface can be obtained from the full RHEED diffraction pattern. Figure 1(a) shows a grazing angle (12 mrad) RHEED diffraction pattern taken along the [110] azimuth of a film grown on a singular substrate at a $5 \mathrm{~Hz}$ laser repetition rate. The curved shadow visible in the lower right of the figure is a Kapton covered
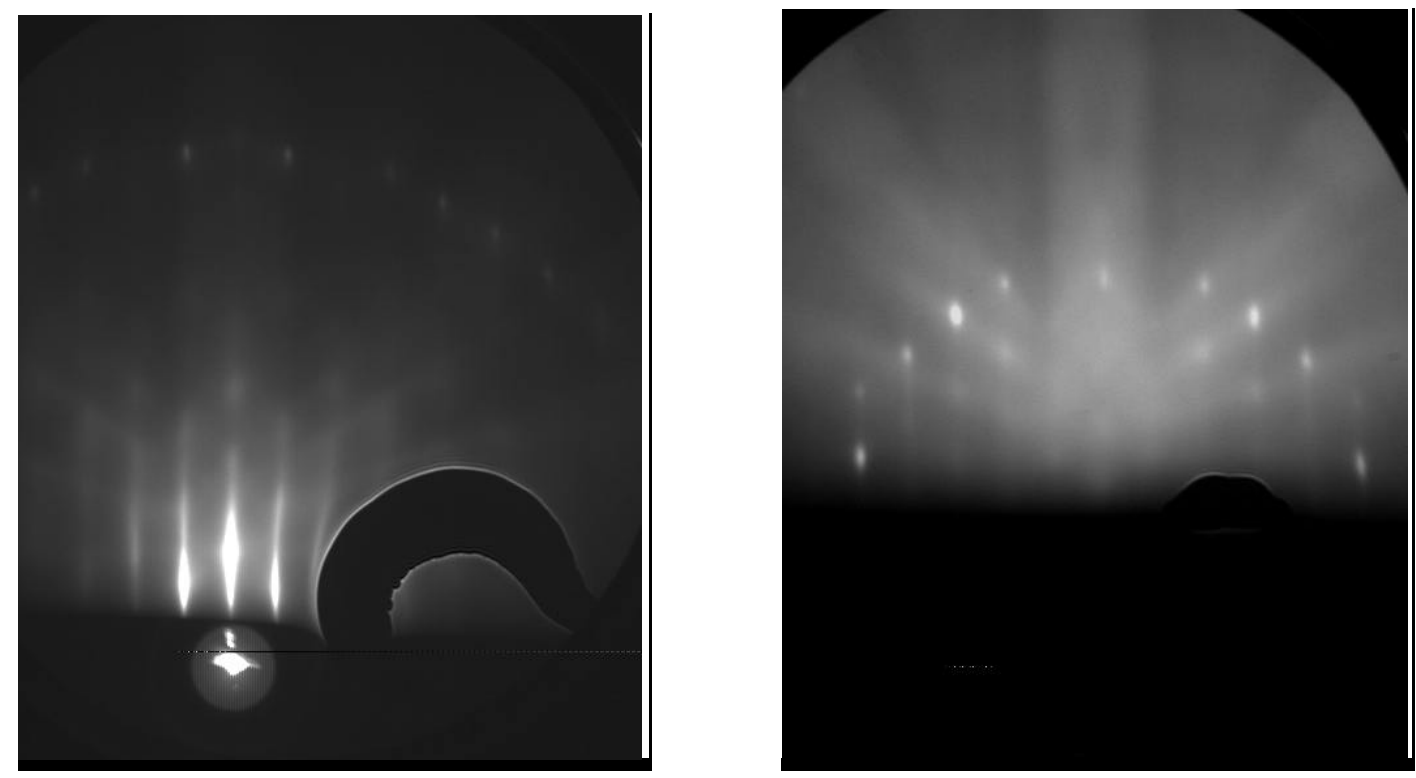

Figure 1. RHEED patterns from the ZnSe thin film grown on singular (001) GaAs at $5 \mathrm{~Hz}$. Both patterns were acquired at $16 \mathrm{keV}$ in the [110] azimuth. Figure 1(a), left, was taken at grazing angle and Figure 1(b), right, was taken at high angle. 
wire attached to a Faraday cup. Figure 1(b) is a pattern from the same sample taken at a higher incident angle $(76 \mathrm{mrad})$. What is seen in the low angle view are streaky diffraction rods from the first Laue zone and sharp spots from the second Laue zone. At the high angle condition, the first zone rods sharpen into spots, while the second zone pattern has shifted off the screen. Other high angle patterns showed strong Kikuchi lines. These originate from the bulk of the film and are indicative of a high quality film with little disorder. No fractional order spots were seen in this or the complementary [1 $\left.1 \frac{1}{0}\right]$ azimuthal geometry. This showed that, in contrast to ZnSe films grown by MBE atomic layer epitaxy [12], the surfaces of those grown by PLD do not reconstruct during growth.

Patterns from both hetero- and homoepitaxial films grown at all laser repetition rates employed (1-5 Hz on singular substrates and $0.5-10 \mathrm{~Hz}$ on the miscut substrates) showed these same features. In an isolated instance, the surface reconstructed when a sample was left in vacuum overnight at room temperature. A film grown on this reconstructed surface grew with an unreconstructed surface and showed the features described above. At no time during or following growth did we see a transmission diffraction pattern which would have indicated the presence of 3 -D structures on the surface.

Figure 2(a) shows the time dependent intensity of the specular spot for a sample grown at $1 \mathrm{~Hz}$ on a singular substrate. It is seen that the intensity of this spot decreases immediately at the start of growth, and reaches a minimum at a film thickness of $\sim 4 \mathrm{~nm}$ (14 bilayers.) The intensity at this point is $5 \%$ above the arbitrarily chosen background signal. The intensity remains at this minimum value throughout the entire growth run. The intensity of the specular spot depends primarily on the morphology of the growth surface. Thus, the decrease in intensity is indicative of a change in surface morphology from one that is favorable for diffraction (e.g., smooth), to one that is not (e.g., islands or increased step edge density); this morphology remains throughout the

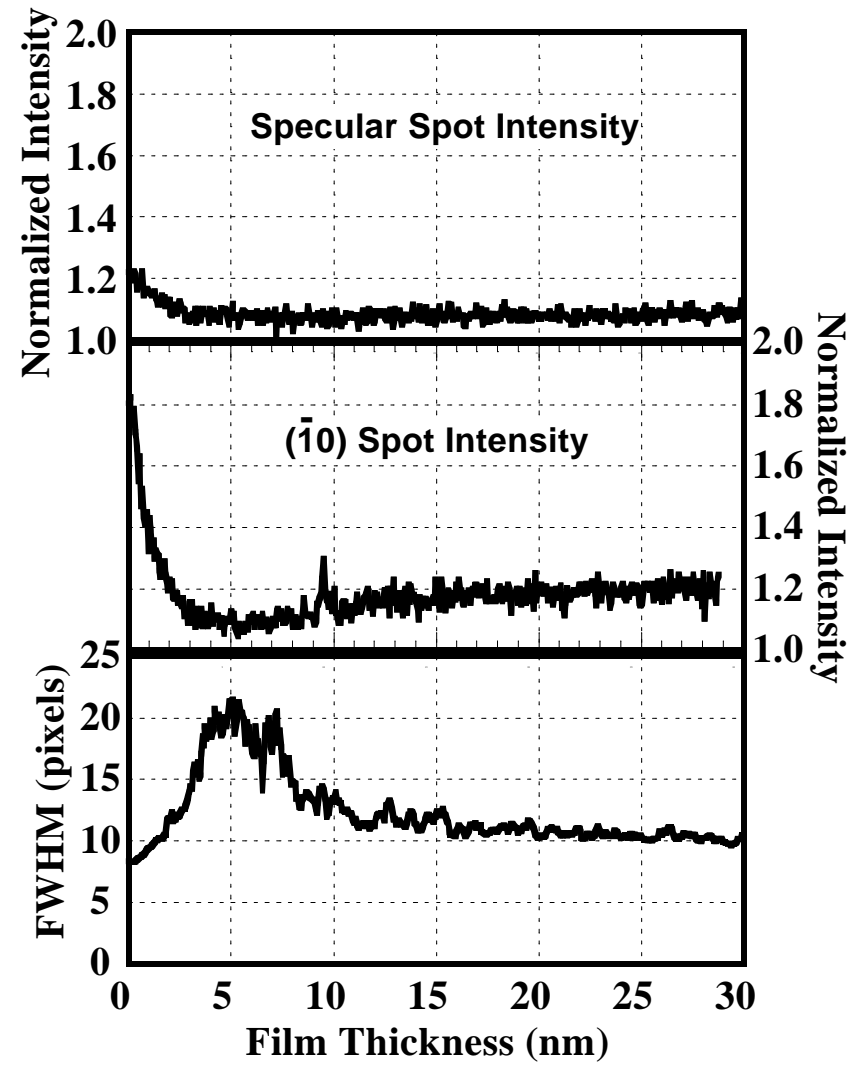

Figure 2. Time dependent RHEED data from a sample grown on a singular substrate at $1 \mathrm{~Hz}$. Figure 2(a), top, is the intensity from the specular spot, Figure 2(b), middle, is the intensity from the (1 0$)$ spot and Figure 2(c), bottom, is the width of the $(\mathbf{1} 0)$ spot. entire growth run.

Figure 2(b) shows the time dependent intensity of the $(\mathbb{1} 0)$ spot, which also reached a minimum at about $4 \mathrm{~nm}$ thickness. However, in contrast to the specular spot, the intensity of this spot stays at the intensity minimum only until a thickness of $7 \mathrm{~nm}$, at which point it increases to a value $10 \%$ above the background. Figure 2(c) shows the width (FWHM) of the ( 10$)$ spot. A broadening of the spot is seen, with the maximum coinciding with the minimum intensity seen in Figure 2(b). The width then decreases sharply until a thickness of $\sim 8 \mathrm{~nm}$ is reached, after which a slower decrease is observed. For non-specular conditions, the diffraction intensity depends strongly on the in-plane order of the growth surface while the width depends inversely on the size of the ordered domains. Thus, the behavior observed is indicative of a decrease in the in-plane order of the growing surface at the beginning of growth, followed by an increase in the order for thicknesses greater than $7 \mathrm{~nm}$.

\section{RESULTS - GROWTH ON VICINAL SURFACES}

Figure 3 shows the time dependent intensity of the specular spot taken along the [1 $1 \frac{1}{0}$ ] azimuth for a sample grown at 


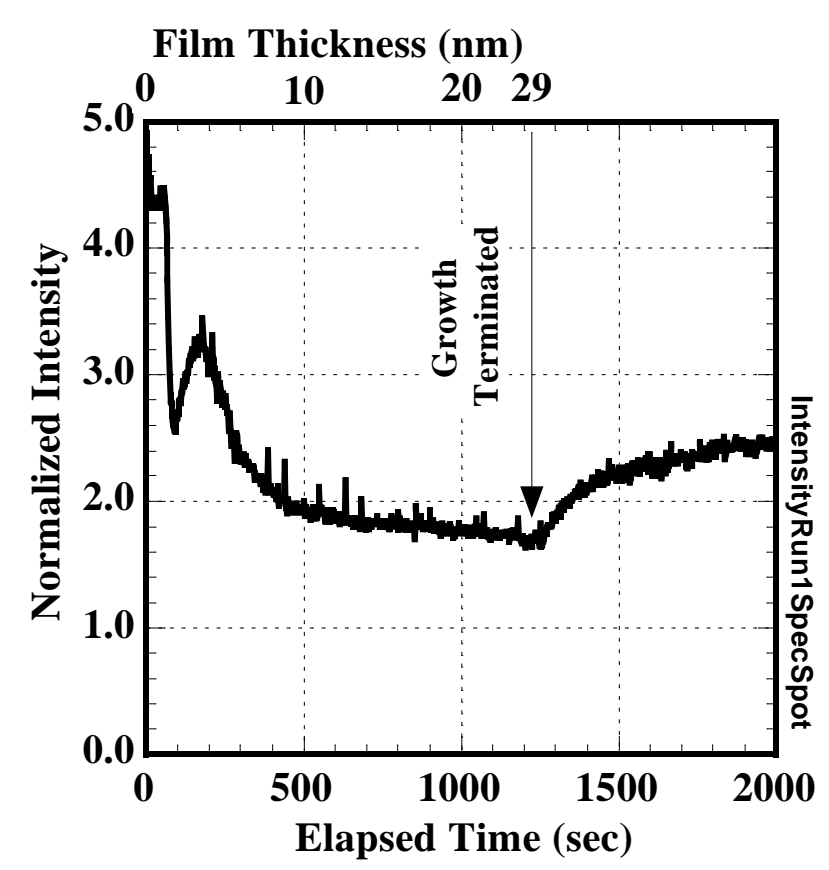

Figure 3. Time dependent intensity of the specular spot taken along the [1 1 ] 0 ] azimuth for a sample grown at $0.5 \mathrm{~Hz}$ on a misalt substrate.

$0.5 \mathrm{~Hz}$ on a miscut substrate. The intensity decreases immediately upon the start of growth, reaching a minimum at a film thickness of $1.7 \mathrm{~nm}$ (6 bilayers). The intensity then increases, reaching a local maximum at $3.4 \mathrm{~nm}$, before decreasing through the rest of the growth run. As noted in the previous section, the intensity of the specular spot depends primarily on the morphology of the growth surface. Thus, as before, the decrease in intensity at the start of growth is indicative of a change in surface morphology from one that is favorable for diffraction to one that is not. Past this local maximum, the intensity decreased in an exponential manner, with an exponent of $-0.32\left(\mathrm{~nm}^{-1}\right)$. The failure of the oscillatory behavior to be maintained indicates that the first morphology is transitory, and that above about $5 \mathrm{~nm}$ a new morphology appears to develop that remains through the rest of the growth run.

When the growth was stopped at a film thickness of $29 \mathrm{~nm}$, recovery of the signal towards a level $50 \%$ below the pregrowth level was seen. This behavior is similar to that seen in MBE growth [13] and is fit by an equation that describes the recovery as having contributions from two processes,

$$
I(t)=A_{0}+A_{1} \exp \left(\frac{-t}{\tau_{1}}\right)+A_{2} \exp \left(\frac{-t}{\tau_{2}}\right)
$$

where the A's are constants, and the $\tau$ 's are the time constants for the slow and fast processes and $t$ is the time measured from the point that growth is stopped. Fitting this equation to the recovery data in Figure 3 yields for the timeonstants: $\tau_{1}=223 \mathrm{~s}$ and $\tau_{2}=26 \mathrm{~s}$, and the ratio $\mathrm{A}_{\mathrm{l}} / \mathrm{A}_{2} \sim 5$.

\section{DISCUSSION}

The appearance in the RHEED patterns of clear, streaky first zone and sharp second zone spots, and well-defined Kikuchi lines is indicative of a smooth growth surface and a high quality film.

For growth on the singular surface, the surface changes from a smooth morphology that is favorable for diffraction to one that is rougher and does not diffract as well. Although this change is easily seen in the time dependent behavior of the specular spot intensity, the exact nature of the morphology cannot easily be inferred from the data obtained here. Comparison of the intensity profile (Figure 2(a)) with that observed during MBE growth leads to the naïve interpretation that the growth occurs in the 3-D island growth mode. However, if this were the case, the surface would be expected to roughen to the point that a transmission diffraction pattern would be observed. This was never seen in this work. Indeed, other work shows that initially rough films smoothen during PLD growth [14]. It is possible that some unique characteristic of PLD, such as the periodicity of the deposition and relaxation processes, or the energetics of the depositing species, limits the increase in roughness. It is possible that the beam energy is also responsible for the lack of reconstruction. This agrees with the work of Yokota, et al., [15] who grew ZnSe films using slightly (2\%) ionized molecular beams. They found that the films grew with a rough morphology if the substrate was not biased, but, when depositing species were made more energetic by biasing the substrate $(-40 \mathrm{~V})$, the films grew with a smoother morphology. Further, 
their published RHEED patterns also show no fractional order spots, indicating that the surface was not reconstructed.

During growth on the singular substrates, the transition towards a steady state morphology is accompanied by a decrease in the in-plane order of the growing surface. This order is recovered as growth continues. The formation of $\mathrm{Ga}$ compounds (e.g., $\mathrm{Ga}_{2} \mathrm{Se}_{3}$ ) at the $\mathrm{ZnSe} / \mathrm{GaAs}$ interface is thermodynamically favored and has been reported [16,17]. Further, it is known that Ga diffusion during the growth of $\mathrm{ZnTe}$ on GaSb leads to autodoping during PLD growth [18]. If this is occurring during the growth of our films, then the presence of $\mathrm{Ga}$ in the region near the $\mathrm{ZnSe} / \mathrm{GaAs}$ interface may be responsible for the observed reduction in in-plane order.

During growth on miscut (001) GaAs at low laser repetition rates $(0.5 \mathrm{~Hz})$, two morphologies form in succession, one transitory and one evolving towards steady state. The intensity profile in Figure 3 is reminiscent of that seen for films grown by MBE primarily in the step-flow mode. However, Gaines and Ponzoni found that the surface diffusion of both $\mathrm{Zn}$ and Se are quite low and that it was not possible to achieve step-flow growth even to a temperature of $440^{\circ} \mathrm{C}$ in $\mathrm{MBE}$ [19]. Although the high kinetic energies of the ablated species may increase the surface diffusion and thereby affect the mode of growth, there is, as yet, no other evidence supporting such a phenomena. Thus, as with growth on the singular surface, it is not possible to determine the mode of growth solely from the data obtained here. We plan to continue this work, supplementing the RHEED data with SPM studies.

The interpretation that there are two relaxation processes following the cessation of growth that result in a recovery of the intensity, is consistent with that observed following MBE growth of GaAs [13, 20]. Based on Monte Carlo simulations Clarke and Vvedensky [21] interpret the fast process as a reduction in the "dendricity" of surface clusters and the slow process as a gross smoothing of the remaining surface. However, in our work, the time constant for the fast process $\left(\tau_{2}=26 \mathrm{~s}\right)$ agrees reasonably well with that determined for the desorption of $\mathrm{Se}\left(\tau_{\mathrm{D}}=30 \mathrm{~s}\right)$ for films grown at this temperature by MBE or atomic layer epitaxy [22,23]. This, coupled with the slow surface diffusivity of both $\mathrm{Zn}$ and Se, suggests an interpretation of the slow process as a reduction in the edge roughness (of either islands or steps), and the fast process as the desorption of Se from the surface.

\section{SUMMARY}

Thin epitaxial films of $\mathrm{ZnSe}$ were grown using PLD on (001) GaAs and $2^{\circ}$ miscut (001) GaAs substrates. In all cases, RHEED patterns following PLD growth showed clear, streaky first zone and sharp second zone spots, and well-defined Kikuchi lines; these features are indicative of a smooth growth surface and high quality film. No reconstruction of the growth surface was observed. Time-resolved RHEED measurements were made and show that a single morphology develops during growth on singular (001) GaAs. However, during growth on miscut (001) GaAs, two morphologies developed, one transient and one steady state. In both cases, comparison of the time-resolved data with that for MBE grown films shows that the mode of growth during PLD does not neatly fall into any of the classical, near-equilibrium descriptions of film growth.

\section{ACKNOWLEDGMENTS}

This research was supported by the U.S. Department of Energy under DE-FG02-89ER45401. We thank Craig Arnold and Jonah Erlebacher for much helpful discussion and a careful reading of the manuscript.

\section{REFERENCES}

1. B.A. Joyce, N. Ohtani, S.M. Mokler, T. Shitara, J. Zhang, J.H. Neave, P.N. Fawceturf. Sci. 298, 399 (1993).

2. J.W. McCamy, D.H. LowndesAppl. Phys. Lett. 63, 3008 (1993).

3. C.M. Rouleau, D.H. Lowndes, J.W. McCamy, J.D. Budai, D.B. Pok,eD.B. Geohegan, A.A. Puretzky, S. Zhu,Appl. Phys. Lett. 67, 2545 (1995). 
4. J. W. McCamy, D. H. Lowndes, J. D. Budai, R.A. Zuhr, Xiao Zhang, Appl. Phys. 73, 7818 (1993).

5. A. Chergui, J.L. Deiss, J.B. Grun, J.L. Loison, M. Robino, R. BesermanAppl. Surf. Sci. 96-98, 874 (1996).

6. J. Simpson, J.O. WilliamsJ. Appl. Phys. 70, 2001 (1991).

7. M. Yoshimoto, H. Nagata, S. Gonda, J.P. Gong, H. Ohkubo, H. Koinumæ,hysica C190, 43 (1991).

8. M.Y. Chern, A. Gupta, B.W. HusseyAppl. Phys. Lett. 60, 3045 (1992).

9. H. Karl, B. Stritzker,Phys. Rev. Lett. 69, 2939 (1992).

10. T. Frey, C.C. Chi, C.C. Tsuei, T. Shaw, F. BozsoPhys. Rev. B 49, 3483 (1994).

11. J.W. McCamy, D.H. Lowndes, J.D. Budai, G.E. Jellison, I.P. Herman, S. Kim, liaser Ablation in Materials Processing: Fundamentals and Applicationstited by B. Braren, J.J. Dubowski and D.P. Norton (Mater. Res. Soc. Proc285, Pittsburgh, PA, 1993) pp. 471-476

12. T. Yao, T. Takeda, Appl. Phys. Lett. 48, 160 (1986).

13. A Yoshinage, M. Fahy, S. Dosanjh, J. Zhag J.H. Neave, B.A. Joyce,Surf. Sci. Lett. 264, L157 (1992).

14. J.W. McCamy, D.H. Lowndes, C.M. Rouleau, unpublished.

15. K. Yokota, S. Tamura, T. Nakajima, S. Katayamal. Cryst. Growth109, 298 (1991).

16. A. Krost, W. Righter, D.R.T. Zanh, O. BrafmanSemicond. Sci. Tech 6, A109 (1991).

17. A.C. Wright, J.O. WilliamsJ. Cryst. Growth114, 99 (1991).

18. D.H. Lowndes, J.W. McCamy and C.M. Rouleau, unpublished.

19. J.M. Gaines, C.A. Ponzoni,J. Vac. Sci. Tech. B10, 918 (1992).

20. S. Clarke, D.D. Vvedensky, M.WRicketts, J. Cryst. Growth95, 28 (1989).

21. S. Clarke, D.D. Vvedensky,Appl. Phys. Lett. 51, 340 (1987).

22. Z. Zhu, M. Hagino,J. Cryst. Growth99, 441 (1990).

23. T. Yao, T. Takeda, J. Cryst. Growth81, 43 (1987). 